\title{
The GenMark ePlex ${ }^{\circledR}:$ another weapon in the syndromic arsenal for infection diagnosis
}

\author{
Jonathan E Schmitz*,1 \& Yi-Wei Tang**,2,3 \\ ${ }^{1}$ Department of Pathology, Microbiology, \& Immunology, Vanderbilt University Medical Center \& School of Medicine, Nashville, TN \\ 37232, USA \\ ${ }^{2}$ Department of Laboratory Medicine, Memorial Sloan Kettering Cancer Center, New York, NY 10065 USA \\ ${ }^{3}$ Department of Pathology \& Laboratory Medicine, Weill Cornell Medical College, New York, NY 10065 USA \\ *Author for correspondence: jonathan.e.schmitz@vanderbilt.edu \\ **Author for correspondence: tangy@mskcc.org
}

\begin{abstract}
As one of the most recent additions to the syndromic testing landscape, the ePlex ${ }^{\circledR}$ platform by GenMark Diagnostics is a system that combines the manufacturer's signature electrochemical detection technology with updated microfluidics, providing a new option for multiplex testing that is both rapid and requires minimal hands-on steps. In this review, we detail the ePlex platform and its current/future syndromic panels, with a particular focus on the respiratory pathogen panel - the platform's first assay to undergo clinical trials and receive regulatory approval in the USA. By keeping informed of these ever-expanding laboratory options, clinicians and microbiologists can stay positioned at the forefront of infectious disease diagnosis.
\end{abstract}

First draft submitted: 17 September 2018; Accepted for publication: 14 November 2018; Published online: 14 December 2018

Keywords: ePlex $\bullet$ GenMark $\bullet$ multiplex molecular testing $\bullet$ respiratory pathogen panel $\bullet$ syndromic infection testing

\section{ePlex ${ }^{\circledR}$ platform}

The care of patients with infectious diseases is increasingly facilitated by technology, in particular laboratory testing, as advanced diagnostic tools can facilitate rapid pathogen-identification and targeted medical management. In recent years, perhaps nowhere have these advances been more prominent than with multiplex molecular assays, which can simultaneously identify and differentiate nucleic acid sequences from diverse infectious agents. Among the most recent multiplex technologies to enter the market is the ePlex ${ }^{\circledR}$ platform by GenMark, this manufacturer's second-generation electrochemical detection system for clinical use (Figure 1). With its Respiratory Pathogen (RP) Panel already widely available - and various other panels in development - the ePlex platform offers new options for clinicians and clinical laboratories. The purpose of this review is to summarize the ePlex platform for these diverse audiences. After providing a brief introduction to multiplex testing, we detail the analytic and diagnostic characteristics of the ePlex platform, with emphasis on the RP panel recent clinical trial data in the USA.

The rise of syndromic molecular testing

Traditional laboratory techniques for diagnosing infections are now frequently complemented with - and, in some cases, replaced by - molecular tests based on the PCR and other nucleic acid amplification techniques. Among the most significant recent advances in diagnostic microbiology are multiplex molecular assays, in which numerous pathogens are targeted in parallel within a single clinical specimen [2,3]. This capability stems from distinct PCR primer/probe sets, combined with detection technologies that can differentially recognize the individual amplicons. These platforms have paved the way for 'syndromic' testing of infectious diseases, allowing a patient with a given set of signs/symptoms (e.g., cough, diarrhea, headache) to undergo a single test for potential causative agents, whose manifestations can demonstrate notorious overlap with one another. These targets can simultaneously encompass viruses, bacteria, fungi and parasites, including pathogens for which previous diagnostic options were limited to esoteric reference tests [4]. 

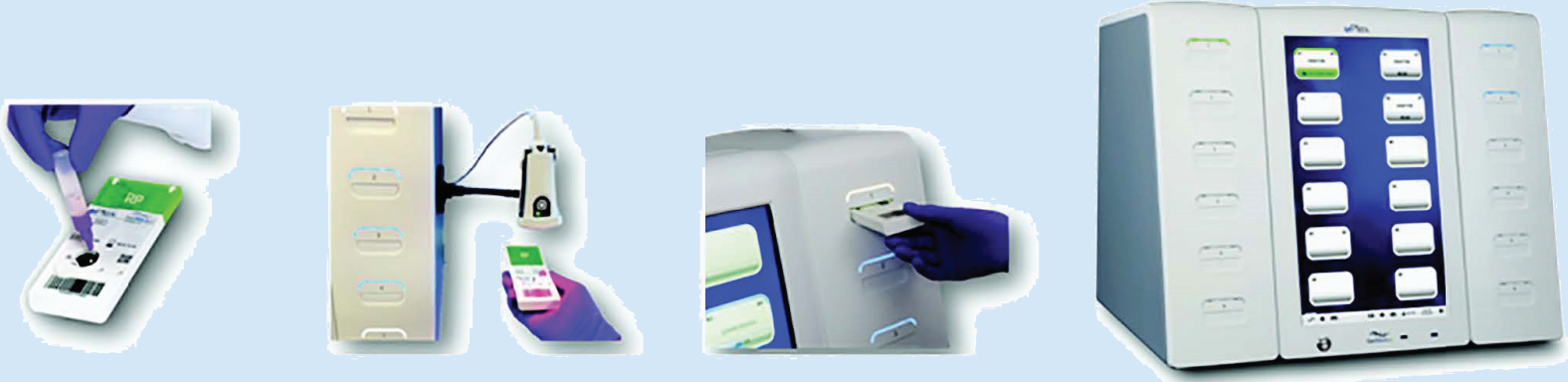

Dispense sample
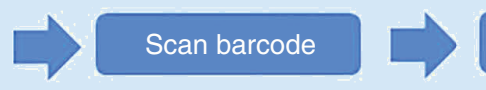

Insert cartridge

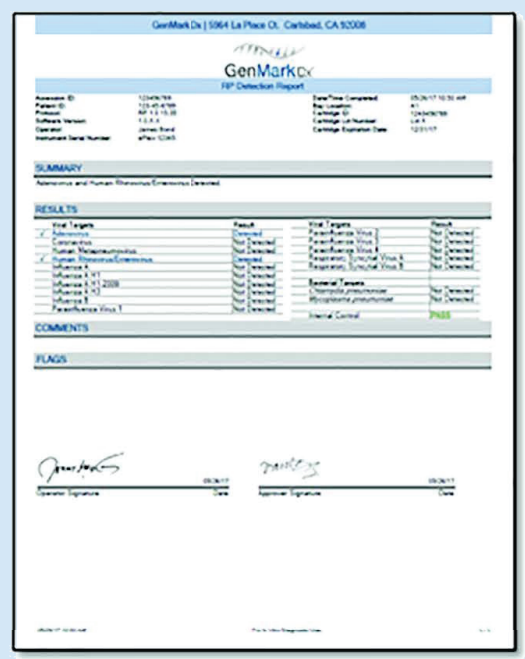

Obtain report

Figure 1. GenMark ePlex ${ }^{\circledR}$ respiratory pathogen panel assay workflow.

Reproduced with permission from [1].

Multiplex assays have significantly altered the clinical approach for many infections, decreasing the time to a definitive microbiologic diagnosis, with downstream benefits to both individual patient-care and antimicrobial stewardship [5]. Admittedly, defining the optimal utilization of these panels is a matter of ongoing reflection across institutions, for instance whether their use should be unrestricted or, rather, limited to particular clinical scenarios and/or specialist services [6,7]. Concomitant financial questions have also emerged, with evolving - in fact, vigorously debated - reimbursement criteria [8]. At the same time, the popularity of these platforms is undeniable and they promise to remain a common fixture in diagnostic labs $[2,3]$.

Syndromic testing has assumed a prominent role for several types of infections given by syndromes' prevalence/severity. Respiratory panels were the first to enter the market with testing performed on nasopharyngeal swab (NPS) specimens. The earliest such assays targeted common circulating viruses, including: influenza A/B, parainfluenza, rhinovirus, adenovirus, respiratory syncytial virus and metapneumovirus [9,10]. Current iterations of respiratory panels now also include several bacterial species with similar clinical features (e.g., Bordetella, Mycoplasma, Chlamydia) [11,12]. Multiplex stool panels were the next to become available with an ability to differentiate among many of the diverse causes of enteritis and colitis (viral, bacterial and parasitic) [13]. Although an explicit species-level diagnosis is often not required for uncomplicated cases of either respiratory or diarrheal illness [14-16], these assays can be incredibly valuable in immunocompromised hosts, as well as in patients with severe 


\begin{tabular}{|c|c|c|c|c|c|c|c|}
\hline \multirow{2}{*}{$\begin{array}{l}\text { Manufacturer } \\
\text { Platform }\end{array}$} & \multicolumn{2}{|c|}{ GenMark } & \multirow{2}{*}{$\begin{array}{l}\text { BioFire } \\
\text { FilmArray }\end{array}$} & \multicolumn{2}{|c|}{ Luminex } & \multirow{2}{*}{$\begin{array}{l}\text { BD } \\
\text { BD MAX }\end{array}$} & \multirow{2}{*}{$\begin{array}{l}\text { T2 Biosystems } \\
\text { T2 }\end{array}$} \\
\hline & ePlex & $\mathrm{XT}-8$ & & Verigene & xTAG/NxTAG & & \\
\hline & $\begin{array}{l}\text { Respiratory } \\
\text { US\&CE IVD }\end{array}$ & $\begin{array}{l}\text { Respiratory } \\
\text { US\&CE IVD }\end{array}$ & $\begin{array}{l}\text { Respiratory }{ }^{\dagger} \\
\text { US\&CE IVD }\end{array}$ & $\begin{array}{l}\text { Respiratory } \\
\text { US\&CE IVD }\end{array}$ & $\begin{array}{l}\text { Respiratory } \\
\text { US\&CE IVD }\end{array}$ & - & - \\
\hline \multirow[t]{3}{*}{ Syndromic panels } & $\begin{array}{l}\text { Blood } \\
\text { CE IVD }\end{array}$ & - & $\begin{array}{l}\text { Blood } \\
\text { US\&CE IVD }\end{array}$ & $\begin{array}{l}\text { Blood } \\
\text { US\&CE IVD }\end{array}$ & - & & $\begin{array}{l}\text { Blood } \ddagger \\
\text { US\&CE IVD }\end{array}$ \\
\hline & $\begin{array}{l}\text { Gl } \\
\text { Development }\end{array}$ & - & $\begin{array}{l}\text { GI } \\
\text { US\&CE IVD }\end{array}$ & $\begin{array}{l}\text { GI } \\
\text { US\&CE IVD }\end{array}$ & $\begin{array}{l}\text { GI } \\
\text { US\&CE IVD }\end{array}$ & $\begin{array}{l}\text { GI } \\
\text { US\&CE IVD }\end{array}$ & - \\
\hline & $\begin{array}{l}\text { CNS } \\
\text { Development }\end{array}$ & - & $\begin{array}{l}\text { CNS } \\
\text { US\&CE IVD }\end{array}$ & - & - & - & - \\
\hline
\end{tabular}

Summarized here are panels for the following major infectious syndromes: respiratory infections, bloodstream infections (e.g., bacteremia/fungemia), gastrointestinal (GI) infections (i.e. diarrheal illness) and CNS infections (e.g., meningitis and encephalitis). The regulatory status of each platform is denoted as: approved for clinical use (IVD) in the USA, approved for clinical use in the European Community (CE), or under development.

†The BioFire FilmArray currently includes an upper respiratory pathogen panel (for nasopharyngeal specimens) available for clinical use in the USA and Europe, as well as a separate pneumoniae panel (for bronchoalveolar lavage specimens) that currently remains under development.

$¥$ With the exception of T2, all other manufacturer's bloodstream panels are performed on positive blood-culture media, whereas the T2 assay is performed directly on whole blood. A candidemia panel for the T2 is IVD-marked in both the USA and Europe, while a bacteremia panel presently remains CE IVD-marked only.

IVD: In vitro diagnostics.

disease or atypical risk factors. More recently, syndromic panels have been developed for less frequently infected anatomic sites - albeit ones with high associated morbidity - with affected patients most often encountered in emergency department and inpatient settings. These include cerebrospinal fluid assays for meningitis/encephalitis [11] and blood-culture assays for bacteremia and fungemia (commonly referred to as 'sepsis' panels) $[17,18]$. The latter differ from other syndromic tests in that multiplex detection is typically performed on microbiological cultures (i.e., initial broth-phase growth in blood culture bottles), not the clinical specimens themselves. More recently; however, the first multiplex tests have emerged for direct-from-blood testing targeting limited bacterial and yeast pathogens [19-22].

It is important to emphasize that syndromic testing is not necessarily limited to prefabricated commercial products, as individual laboratories (either single-institution labs or reference facilities) can and do develop multiplex molecular assays for in-house use ('home brews') [23,24]. At the same time, the inherent complexity of these tests - and the effort and cost needed to validate them rigorously - make syndromic assays conducive to commercial development, with formal approval by national regulatory bodies and large-scale distribution. This approach has become the dominant paradigm in clinical practice, as the diagnostic market currently entails several major manufacturers competing through their respective proprietary assays. These technologies each constitute a central detection platform (i.e., the instrument itself), which is then interfaced with separate panels for the various syndromes (e.g., a respiratory panel, stool panel, etc.).

With GenMark, for instance, pathogen detection involves ferrocene-labeled oligonucleotide probes, which associate with electrodes via amplicon-probe annealing events, altering impedance in a measurable way (as detailed below). Other major manufactures offering syndromic panels include: the FilmArray by BioFire (parent company bioMerieux), which utilizes nested PCR in sequential microreservoirs, with downstream fluorescence-detection on a $2 \mathrm{D}$ grid $[11,13,17,18,25,26]$; the VERIGENE by Luminex, which detects amplicons through gold/silver-conjugated oligonucleotide probes and an evanescent field phenomenon [17,18,26]; the xTAG and NxTAG flow cytometry systems, also by Luminex, which employ fluorescently barcoded microbeads to target amplicons [12,26,27]; the BD MAX system by BD, a qPCR platform that enables multiplexing through automated robotics [28,29]; and the T2Dx platform by T2 Biosystems, which utilizes NMR relaxometery to detect amplicon-mediated agglomeration of capture probes [19,21]. Additional technical details and performance characteristics of these platforms are detailed in the respective citations.

\section{Syndromic testing platforms: demand shaping design}

From these options, a clinical laboratory must determine which technology best addresses its local needs. The above platforms currently include panels for one or more of the major syndromes, as summarized in Table 1 (classified as US approved, European Community approved or under development). Not surprisingly, the available panels from each manufacturer are increasing with time, allowing a laboratory to maximize the testing that can be accomplished through a single platform. This flexibility can be quite attractive from an operational and financial perspective, as 
Table 2. Pathogen targets on various multiplex respiratory panels.

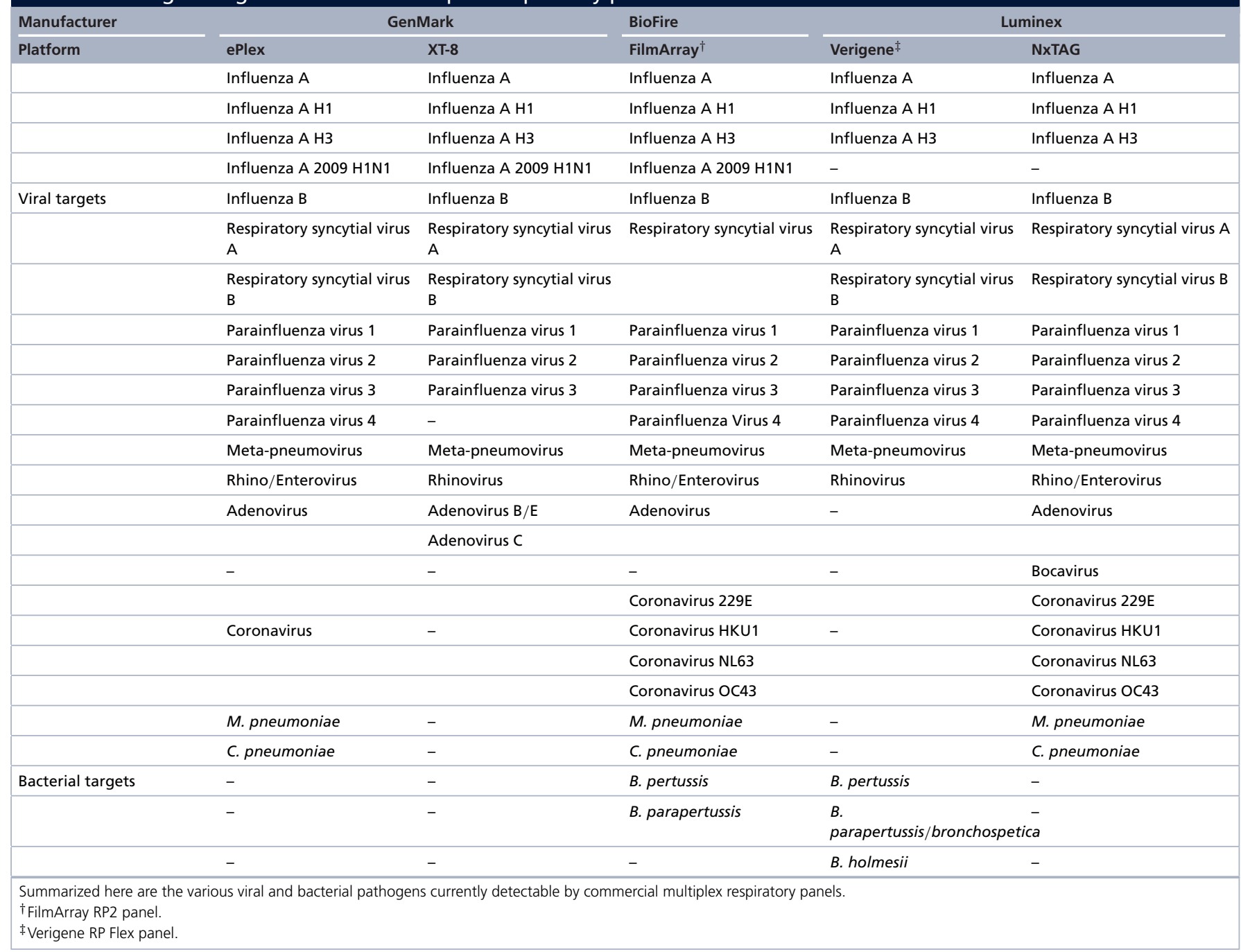

the platforms themselves typically represent 'big-ticket' items for an institution, entailing a capital purchase or lease agreement. In addition to multipanel capability, a variety of other considerations define a platform's overall utility along with its potential limitations - and several of these clinical, analytical and logistical factors are discussed here.

On a practical level, a multiplex assay is valuable insofar as it can generate a rapid and accurate identification among the numerous pathogens on a differential diagnosis. In this regard, one of the most common selling points for a system is the number of different agents that it is capable of detecting (i.e., its extent of multiplexing). The targets of commercially available respiratory panels, for instance, are summarized in Table 2 . Across manufacturers, an ongoing trend involves the development of panels that incorporate a greater number of targets. In fact, compared with respiratory pathogens, the diversity of diarrheagenic pathogens is even greater, with a large variety of uncommon agents alongside the more frequent etiologies. And for specimens like blood and cerebrospinal fluid, the number of organisms that might theoretically cause infection is truly vast, especially when opportunistic agents and uncommon risk factors are considered. The ability of an assay to diagnose both common and uncommon pathogens has obvious clinical desirability, especially considering that rare infections are often associated with significant diagnostic latency.

At the same time, several caveats also apply to syndromic assays with large-scale multiplexing. To begin, one must recognize the fundamental relationship between the specificity of a test, its positive predictive value, and the prevalence of the targeted disease: even tests with relatively high specificity can demonstrate a low positive predictive value for uncommon infections. With diarrheal illness, in particular, there are a number of organisms that can be associated with both symptomatic infection and asymptomatic colonization (e.g., enteropathogenic 
and enteroaggregative Escherichia coli [30-32] and Clostridium diffcile [33,34]). When such organisms are included on a panel, a positive result does not necessarily imply a causative role in the patient's symptoms, especially when multiple targets are detected in the same specimen [35]. Finally, at the intersection of laboratory finances and diagnostic stewardship, the 'more-is-better' paradigm for syndromic targets must balance the cost of testing with the specific needs of the individual. An assay with additional targets might entail greater expenses for the performing laboratory, the insurance/payer or the patient him/herself, and these factors must be weighed in light of the pretest probability of the various pathogens and the overall goals of care. These issues are reflected by the recent development of the first panel (on the VERIGENE system) for which the resulted targets are selected on a specimen-by-specimen basis, with the cost of testing adjusted accordingly [36].

Alongside the issue of which targets a multiplex assay can detect is the vital question of how fast this testing can occur. The logistics of turnaround time - from when a specimen is collected to when results are available - are always at the forefront of laboratory operations, and this dynamic certainly holds true for syndromic testing. For many scenarios, in fact, a rapid result is precisely what allows a multiplex assay to impact patient care (e.g., through earlier escalation/deescalation of therapy or the real-time decision to admit/discharge). Just as recent syndromic panels include a growing number of targets, so too has there been an emphasis on designing platforms that generate results as rapidly as possible. The time required for the individual steps of nucleic acid extraction, PCR amplification and amplicon detection are contributing factors, although an even greater determinant of turnaround time is a platform's ability to connect these steps in an automated fashion. The first generation of syndromic assays employed separate extraction and amplification steps, followed by manual transfer of amplicons onto the platform for detection $[26,27,37]$. More recently, however, panels have been engineered as self-enclosed cartridges that house extraction, amplification and detection steps in discrete physical locations, linked to one another through microfluidic channels. After a specimen is loaded onto the cartridge, it is interfaced with the platform and the entire analytic process proceeds sequentially. This hands-off capability (currently available with the ePlex, FilmArray and VERIGENE systems) eliminates delays from manual transfers [11,13,17,18,25,26]. Just as significantly, the design allows specimens to be analyzed on a random-access basis as they arrive in the laboratory, eliminating the need for batched testing and the limitations this places on achieving on consistent turnaround times.

Beyond these considerations, self-enclosed testing cartridges afford several other benefits to laboratory workflow and quality assurance. As with any nucleic acid amplification test, amplicon carryover (i.e., cross-contamination from one specimen to the next) remains a prominent concern with multiplex systems, further magnified by the number of separate amplicons that might act as contaminants [38]. To address this concern, a self-enclosed design - where amplicons are never manipulated or exposed to the external environment - significantly mitigates the opportunities for carryover and the adverse clinical consequences of false-positive results. It likewise reduces an assay's technical complexity for laboratory staff and the need for technologists with specific expertise in molecular biology. In fact, when combined with rapid turnaround times, operator-friendly platforms create an opportunity for syndromic testing outside of centralized laboratories. Recently, the first multiplex test was approved by the US FDA as a CLIA-waived assay (the FilmArray EZ respiratory assay), opening the door for point-of-care testing in outpatient clinics, emergency department and hospital wards [39]. Admittedly, there is still a need to further delineate the specific circumstances (both clinical and financial) in which multiplex point-of-care testing is most appropriate and impactful $[40,41]$. Nonetheless, the development of such instruments highlights the factors that are currently driving the syndromic testing market.

Along with this focus on target-coverage and turnaround time, multiplex platforms are incorporating additional features to benefit laboratory and clinical workflow. With a random-access design, it is critical that platforms can interface with multiple independent cartridges for simultaneous testing. An instrument's optimal number of testing bays; however, depends on a given institution's clinical volume. Manufactures now commonly offer platforms in scalable configurations that can meet these diverse needs. Across different instrument configurations, features like barcode scanning and touchscreen graphical displays can further simplify the efforts of laboratory staff. Although syndromic assays are increasingly designed for ease-of-use, their results often carry nuanced implications for patient care, especially when less common targets are encountered. Additional interpretative guidance (crafted jointly by laboratory and clinical stakeholders) can add further value to the basic findings of detected or not detected. 


\section{The eplex platform}

\section{Basic features of the instrument}

Against this complex backdrop of clinical and logistical needs, the ePlex platform has recently entered the diagnostic market in the USA and internationally. As mentioned above, the ePlex platform is GenMark's second-generation multiplex platform, following the XT- $8{ }^{\circledR}$ instrument whose Respiratory Viral Panel (RVP) was launched in $2012[42,43]$. The ePlex platform was launched with a specific focus on syndromic infection testing and a sample-toresult workflow. As depicted in Figure 1, the platform incorporates a scalable number of towers (from 1 to 4), each of which houses bays for six independent tests [44]. With an analysis time of $<2 \mathrm{~h}$ per assay, these configurations can support a throughout of 24-96 tests within a typical 8-h shift. Although these larger towers are intended for central laboratory usage, an additional ePlex instrument configuration - the NP ('near patient') - is available with three bays. The ePlex NP is an option for institutions running as few as 12 samples per shift. The basic external features of an ePlex cartridge include a port for specimen input, a unique barcode for cartridge identification, and an open area for placing a specimen accession ID barcode. Each self-enclosed cartridge contains all the regents and components of the test. Cartridges require refrigeration prior to use, but require minimal manipulation by laboratory staff during testing. In the case of the RP Panel, $200 \mu$ of a NPS specimen (in liquid transport media) is transferred into the sample delivery device and vortexed for $10 \mathrm{~s}$. The entire volume of the sample delivery device is dispensed into the cartridge, entailing $\sim 2$ min of hands-on time. Nucleic acid is extracted within the cartridge with automated fluidic transport to the amplification and detection steps. Other features of the ePlex platform likewise place an emphasis on ease-of-use and rapid turnaround time (addressing the general points discussed above). These include characteristics of both the instrument itself and its software, as summarized by the product literature from the manufacturer [45].

\section{eSensor electrochemical detection}

Before describing the available panels and performance characteristics of the ePlex platform, we first wish to discuss the underlying technology that makes the analysis possible. The central design feature of both the XT- 8 and the ePlex platform is a proprietary method of electrochemical nucleic acid detection called eSensor technology. This method exploits a basic biophysical phenomenon that impedance across an electrical circuit can be modulated by redoxactive metalloorganic moieties. Specifically, the eSensor technology utilizes an oligonucleotide 'sandwich' approach to recruit ferrocene derivatives to the surface of gold-plated electrodes. A detailed schematic and video of the process is available [46]. After PCR thermocycling, an exonuclease digestion is performed to create single-stranded amplicons; these amplicons are first annealed to ferrocene-conjugated signal probes. The resultant complexes maintain an amplicon-overhang that is complimentary to separate capture probes, which are bound to a gold electrode [42]. The signal probe-amplicon complexes bind to the capture probes, immobilizing ferrocene at the electrode as part of a three-nucleotide complex. With this assembly, iron-redox cycling is induced by an applied potential at the electrode, and the resultant faradaic current is assessed voltammeterically against the background capacitative current. By probing this relationship at each electrode, one can rapidly asses which amplicons (and source pathogens, in turn) are present or absent [42].

The eSensor technology was originally developed in 1993 at the California Institute of Technology by Jon Faiz Kayyem. This technology was later commercialized and the initial use of electrochemical detection of DNA on a ship was focused on diagnosing human genetic diseases that included hereditary hemochromatosis and cystic fibrosis [43]. Corporate ownership of the technology has changed hands several times in subsequent years, and genetic testing on the XT-8 remains a valuable component of the eSensor technology's overall diagnostic utility. Nevertheless, an expansion to syndromic applications is not surprising, as the eSensor technology's inherent designstrengths are highly conducive to these purposes. The technology allows for a high degree of multiplexing, as the maximum number of targets in a panel is a function of the working electrodes in its circuit board. Diagnostic specificity, moreover, is improved by the need for multiple oligonucleotide annealing to generate an electrochemical signal. The speed at which amplicons are detected, the brief time required to sweep a voltage across individual electrodes, lends itself to a rapid diagnostic turnaround.

\section{Integrated electrowetting fluidics}

Despite these strengths of the eSensor technology, a shortcoming of the XT-8 platform for syndromic testing is the aforementioned need to extract specimens and transfer amplicons manually. The sample-to-result capability of the ePlex platform is one of the most important upgrades to the eSensor technology, achieved through integration with 
a microfluidic phenomenon known as electrowetting on dielectric. This pump/valve-free technology employs a hydrophobically coated dielectric material on top of individual metallic imbedded electrodes [1]. When microlitervolume aqueous specimens are located on the surface, the droplets can be forced to migrate along a desired route by applying voltages sequentially at contiguous electrodes. This electrical potential decreases the local interfacial tension between the liquid and the plate's surface, inducing the droplet to align with the activated electrode. The resulting 2D pathway allows a liquid specimen to be introduced in a step-wise manner to the various reagents of a PCR assay (for extraction, amplification and detection), prefabricated on the surface at the necessary locations. Purification and concentration of nucleic acids are facilitated by paramagnetic beads, magnetically immobilized at specific locations of the pathway. During target amplification, a reaction mixture can be actuated back-and-forth over appropriately heated surface-locations, facilitating rapid in-droplet thermocycling. In total, an ePlex cartridge represents an electrowetting-mediated PCR module interfaced with an eSensor device for final amplicon detection. The combined technology was facilitated by a partnership between GenMark and Advanced Liquid Logic (acquired by Illumina ${ }^{\circledR}$ ), where the microfluidic process was developed.

\section{Diagnostic performance of eplex platform}

Overview \& current status of ePlex panels

As with all commercial diagnostics, the availability of a syndromic assay is ultimately dictated by its regulatory approval in a particular market. Accordingly, the RP panel is the first ePlex panel to receive marking as an in vitro diagnostic within both the European Community (2016) [47] and the USA (2017) [48], the latter via 510(k) FDA clearance (with the FilmArray Respiratory Panel as the predicate device). The ePlex panel covers 15 viral and 2 bacterial pathogens, already summarized in Table 1. NPS in viral transport medium are the FDA cleared, validated specimen types, as other specimen types (e.g., bronchoalveolar lavage fluid, tracheal aspirates) have not been evaluated in clinical studies. It is important to note that the latter specimen-types are not covered under the FDA clearance of the ePlex RP panel, and a US laboratory would be required under the Clinical and Laboratory Improvements Amendments to conduct additional validation studies on-site prior to implementing clinical testing. Although this same situation currently applies to all commercial respiratory panels (with NPS-only clearance), it still represents a source of potential confusion. Clinicians often desire multiplex testing of alternative respiratory specimens, and the medical literature has included various examples of such off-label use (for various manufacturer's platforms) [49-52]. Importantly, whenever a new platform comes to market, the performance of a nonapproved specimen type cannot simply be assumed and must be demonstrated explicitly, per local regulatory mandates.

Among the individual targets on the ePlex RP panel, several merit brief discussion for how their detection compares with other commercial syndromic assays. As one of the most epidemiologically significant respiratory pathogens, influenza is targeted by the ePlex RP platform with a similar strategy to the other current platforms [11]. The RP panel detects influenza types A and B independently through separate assays, while additional subtype assay $(\mathrm{H} 3, \mathrm{H} 1$ and $2009 \mathrm{H1})$ define the lineage within type A. Novel/atypical influenza strains would be indicated by a type A-only detection (without any subtypes), although this rare - and potentially concerning - event would require further confirmation by public-health authorities. Among the most common detections, the ePlex RP panel reports rhinovirus and other enteroviruses together (as rhinovirus/enterovirus). Although differs from the rhinovirusspecific probe of XT-8 panel, several other platforms utilize this same strategy [25], in which other enteroviruses are not detected. Also, unlike the XT-8 system, the ePlex RP panel includes coverage of several corona viruses (229E, HKU1, NL63, OC43), although it does not differentiate between them in its final report ${ }^{1}$. The CE-marked version of the ePlex RP Panel includes analytical data for Middle East Respiratory Syndrome Coronavirus; however, this target is not available on the IVD cleared assay. Finally, the ePlex RP Panel includes Mycoplasma pneumoniae and Chlamydia pneumoniae among its bacterial targets, although it does not detect any Bordetella species on the IVD cleared panel (covered in several other commercial panels [11]).

The ePlex blood-culture Identification panels have likewise received CE-IVD marking in the European Community (in 2017): a Gram-positive (GP) panel, Gram-negative (GN) panel and a fungal pathogen (FP) panel [53]. In each case, testing is performed on blood-culture media in which microbiologic growth has already been detected by standard fluorometeric monitoring techniques. The choice of which panel to utilize (GP, GN or fungal) is dictated by the initial Gram-stain results of that specimen. This approach is similar to current VERIGENE blood culture panels (which include GP and GN assays, but not fungi), although it differs from the FilmArray panel (in which bacterial and fungal targets are combined into a single assay) [17]. As with the ePlex RP panel, the blood-culture 
Table 3. Pathogen targets on the ePlex blood culture panels.

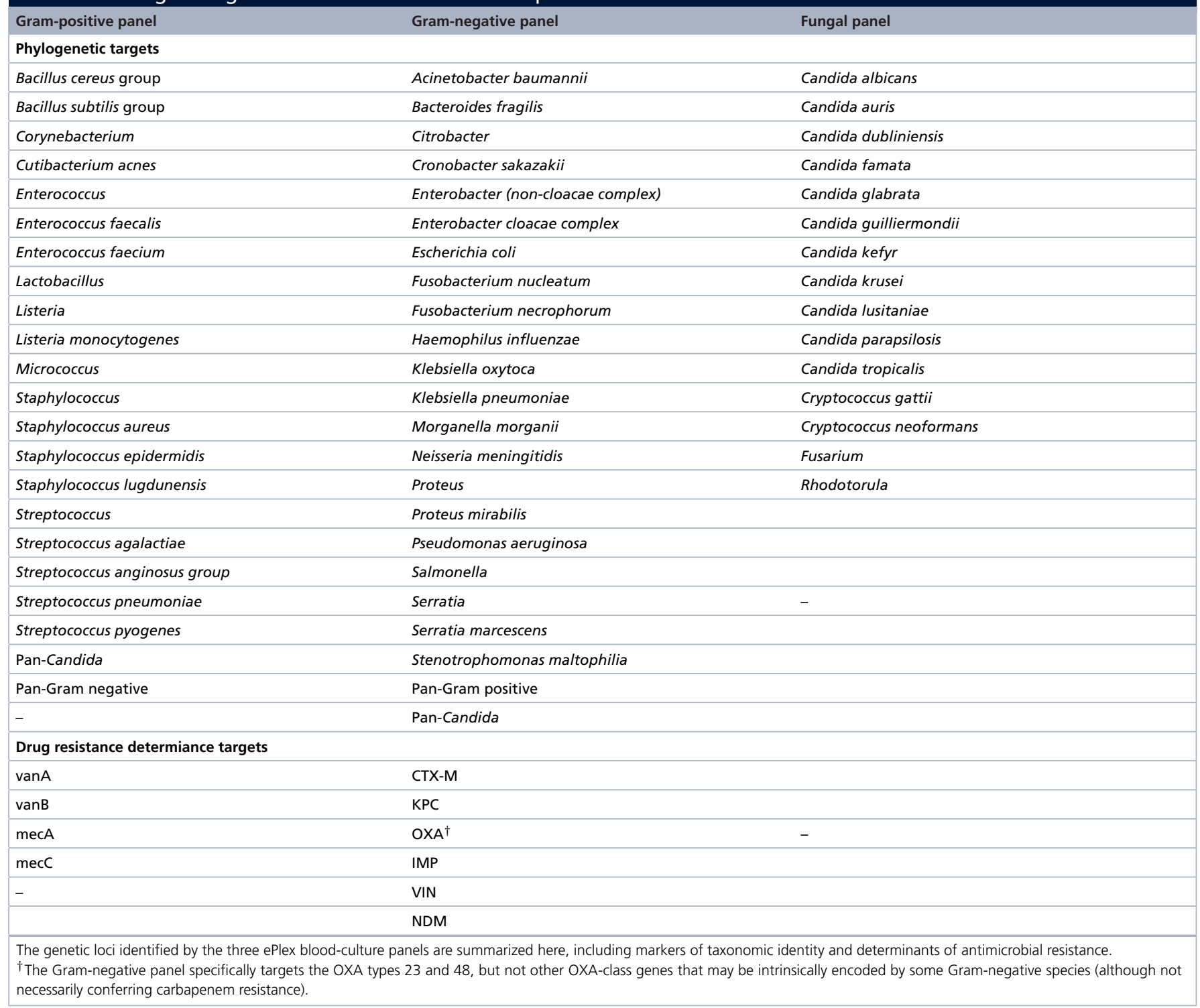

media is loaded directly into the port of the respective cartridge, without a need for manual preprocessing. Extraction, amplification and detection all proceed in an automated fashion within the cartridge, with a total analysis time of $\leq 2 \mathrm{~h}$. Unlike the VERIGENE blood-culture panels, which directly fragment the bacterial DNA without target amplification, a dedicated PCR step is part of the ePlex methodology. Accordingly, the ePlex platform affords greater flexibility in terms of when - and after how many freeze/thaw cycles - a blood culture bottle can be analyzed (or re-analyzed) following initial detection of growth.

The specific targets of the ePlex Blood-Culture Identification panels are summarized in Table 3, compared with the targets of other commercial assays. The GN- and GP-panels include a number of genus- and/or species-level taxonomic probes, in addition to several probes for key antimicrobial resistance determinants, while the fungal panel provides taxonomic information only. The ePlex bacterial panels also include a pan-GP or pan-GN probe, as well as a pan-Candida probe. These serve as valuable quality controls, in the event that the preceding Gram stain was misread or demonstrated an atypical staining pattern, for instance leading to a GN organism being loaded onto a GP panel (or vice versa). As evident in Table 3, one of the most noteworthy features of the ePlex panels is that additional organism coverage they provide over other commercial options. These include a variety of common skin contaminants for the GP panel, additional nonfermenter and anaerobic coverage on the GN panel, and several 
non-Candida fungal species on the fungal panel, including rarer agents of potentially severe opportunistic infections that can demonstrate intrinsic or acquired antifungal resistance [53]. Clinical trial data have not yet been published on the ePlex blood culture panels, although these studies have been completed. Data for the three panels were recently submitted to FDA for review [54].

\section{Respiratory clinical trials}

To date, the most extensive published evaluation of an ePlex panel has been the North American clinical study of the RP panel, constituting a significant portion of the assay's FDA submission data [1]. A multicenter clinical trial was performed to assess the clinical performance characteristics of the ePlex RP Panel for the detection of viral and bacterial respiratory tract pathogens in NPS specimens collected at 13 sites across the USA and Canada [1]. Prospectively and retrospectively collected NPS specimens were evaluated by using the bioMérieux/BioFire FilmArray Respiratory Panel (BioFire RP) as the comparator method. Discordant analysis was performed by using target-specific PCRs and bidirectional sequencing. Of the 3235 samples eligible for testing with the ePlex RP panel, 154 had invalid results (4.8\%). After repeat testing, eight samples were not evaluable due to final, invalid results. The final validity rate was $99.8 \%$.

The overall agreement was more than $95 \%$ for all targets between the ePlex RP and BioFire RP panels. In comparison with the BioFire RP, the sensitivities for viruses ranged from $85.1 \%$ (95\% CI: 80.2-88.9\%) to 95.1\% (95\% CI: 89.0-97.9\%), while specificities ranged from 99.5\% (95\% CI: 99.1-99.7\%) to 99.8\% (95\% CI: $99.5-$ $99.9 \%)$. Additional testing of discordant targets (12\%; 349/2908) confirmed the results of ePlex RP for $38 \%$ $(131 / 349)$ of samples tested. The reproducibility of the assay was evaluated at three sites by using a reproducibility panel which consisted of six targets. Reproducibility was $100 \%$ for all targets tested, with the exception of adenovirus, for which reproducibilities were $91.6 \%$ at low virus concentrations and $100 \%$ at moderate virus concentrations [1].

In addition to assay performance, other characteristics, including assay workflow and turnaround time, were evaluated. The ePlex instrument consists of a stand-alone system and a touch screen, with an option for spaceconstrained laboratories. The ePlex instrument is available in configurations ranging from one to four towers, with each tower containing six random-access testing bays; the NP configuration designed for low-volume sites has three bays in a single tower. This supports single-shift $(8 \mathrm{~h})$ testing volumes ranging from 24 to 96 samples, with a turnaround time of approximately $2 \mathrm{~h}$ and a hand-on time of less than $2 \mathrm{~min}$, providing a flexible capacity for laboratories with variable sizes. The ePlex reagents need to be stored in refrigerator upon arrival [1].

Two additional clinical studies have been reported in Europe. van Rijn et al. clinically validated the ePlex RP Panel in comparison to a laboratory-developed test (LDT). A total of 30 specimens tested LDT positive (47\%) and $29 \mathrm{ePlex}{ }^{\circledR} \mathrm{RP}$ panel positive (45\%). The median time to result was $3.4 \mathrm{~h}$ (range 1.5-23.6) for the ePlex RP panel [52]. In another study, the ePlex RP panel was evaluated on clinical specimens using LDT real-time PCR assays as referees. The RP panel showed an agreement of $97.4 \%$ with the LDT regarding 464 pathogens found in the clinical specimens. The RP panel detected 17 additional pathogens seven of which could be confirmed by discrepant testing [50].

\section{Future directions}

Looking to the future, GenMark has announced several additional panels that are currently under development for the ePlex platform [55], although they have not received CE-IVD marking in Europe or the IVD clearance in the USA and product literature is not yet publicly available. These include a CNS panel for meningitis and encephalitis, and a gastrointestinal pathogen panel for diarrheal illness. Once these panels are brought to market, the syndromes covered by the ePlex platform will be commensurate to all other available commercial platforms. Although GenMark has not announced the specific targets for these assays, clinical laboratories will view with great interest how they compare with current options. Finally, while not a syndromic test per se, mirroring current availability of this analysis on the XT-8 system, a multiplex assay for HCV genotyping has also been announced for the ePlex pipeline.

\section{Financial \& competing interests disclosure}

This study was funded in part through NIH/NCI Cancer Center support grant P30 CA008748 and an agreement between GenMark and Memorial Sloan Kettering Cancer Center. The authors have no other relevant affiliations or financial involvement with any organization or entity with a financial interest in or financial conflict with the subject matter or materials discussed in the manuscript apart from those disclosed. 


\section{Company Review}

In addition to the peer-review process, with the author's consent, the manufacturer of the product discussed in this article was given the opportunity to review the manuscript for factual accuracy. Changes were made by the author at their discretion and based on scientific or editorial merit only. The author maintained full control over the manuscript, including content, wording and conclusions.

\section{Executive summary}

Recent years have witnessed a paradigm shift in the laboratory diagnosis of infectious diseases

- Development and implementation of multiplex molecular assays to simultaneously detect nucleic acid from various pathogens (bacterial, viral, fungal and/or parasitic) within a single clinical specimen.

- This ability for culture-free, syndromic testing has facilitated more rapid and comprehensive diagnoses, driven in large part by several commercial technologies whose combined presence is increasingly ubiquitous in clinical laboratories.

The ePlex platform represents one of the most significant new additions to the syndromic testing landscape

- The ePlex instrument consists of a stand-alone system, with no additional accessories and a touch screen, making it a great option for space-constrained laboratories.

- The ePlex instrument is available in configurations ranging from one to four towers, with each tower containing six random-access testing bays.

- This supports single 8-h shift testing volumes ranging from 24 to 96 samples, with a turnaround time of less than $2 \mathrm{~h}$ and a hand-on time of less than $2 \mathrm{~min}$, providing a flexible capacity for laboratories of all sizes.

It is predicted that the ePlex platform meets the diverse needs encountered in clinical practice

- A number of other panels undergoing trials and development.

- This platform and its associated panels will become an increasingly common sight in diagnostic laboratories with an ability to significantly impact patient care.

\section{References}

Papers of special note have been highlighted as: $\bullet$ of interest; $\bullet \bullet$ of considerable interest

1. Babady NE, England MR, Jurcic Smith KL et al. Multicenter evaluation of the ePlex respiratory pathogen panel for the detection of viral and bacterial respiratory tract pathogens in nasopharyngeal swabs. J. Clin. Microbiol. 56(2), pii:JCM.01658-01617 (2018).

-. This was the publication reporting that the first multicenter clinical trial results on the GenMark ePlex RP panel.

2. Hanson KE, Couturier MR. Multiplexed molecular diagnostics for respiratory, gastrointestinal, and central nervous system infections. Clin. Infect. Dis. 63(10), 1361-1367 (2016).

3. Ramanan P, Bryson AL, Binnicker MJ, Pritt BS, Patel R. Syndromic panel-based testing in clinical microbiology. Clin. Microbiol. Rev. 31(1), pii:31/31/e00024-00017 (2018).

- A nice comprehensive review on syndromic panel-based testing for infections.

4. American Society for Microbiology . Multiplex White Paper: Clinical Utility of Multiplex Tests for Respiratory and Gastrointestinal Pathogens (2018). www.asm.org/index.php/statements-and-testimony/item/6691-wp-multiplex

5. Messacar K, Parker SK, Todd JK, Dominguez SR. Implementation of rapid molecular infectious disease diagnostics: the role of diagnostic and antimicrobial stewardship. J. Clin. Microbiol. 55(3), 715-723 (2017).

6. Dien Bard J, Alby K. Point-counterpoint: meningitis/encephalitis syndromic testing in the clinical laboratory. J. Clin. Microbiol. 56(4), pii:JCM.00018-00018 (2018).

7. Schreckenberger PC, McAdam AJ. Point-counterpoint: large multiplex pcr panels should be first-line tests for detection of respiratory and intestinal pathogens. J. Clin. Microbiol. 53(10), 3110-3115 (2015).

- A nice point-counterpoint article covering pros and cons of large multiplex PCR panels in clinical microbiology practice.

8. American Society for Microbiology. Multiplex White Paper: Clinical Utility of Multiplex Tests for Respiratory and Gastrointestinal Pathogens (2018). www.asm.org/index.php/statements-and-testimony/item/6708-pal-test

9. Krunic N, Yager TD, Himsworth D, Merante F, Yaghoubian S, Janeczko R. xTAG RVP assay: analytical and clinical performance. J. Clin. Virol. 40(Suppl. 1), S39-S46 (2018).

10. Merante F, Yaghoubian S, Janeczko R. Principles of the xTAG respiratory viral panel assay (RVP Assay). J. Clin. Virol. 40(Suppl. 1), S31-S35 (2007)

11. Leber AL, Everhart K, Daly JA et al. Multicenter evaluation of BioFire FilmArray respiratory panel 2 for detection of viruses and bacteria in nasopharyngeal swab samples. J. Clin. Microbiol. 56(6), pii:JCM.01945-01917 (2018).

12. Tang YW, Gonsalves S, Sun JY et al. Clinical evaluation of the Luminex NxTAG respiratory pathogen panel. J. Clin. Microbiol. 54(7), 1912-1914 (2016). 
13. Khare R, Espy MJ, Cebelinski E et al. Comparative evaluation of two commercial multiplex panels for detection of gastrointestinal pathogens by use of clinical stool specimens. J. Clin. Microbiol. 52(10), 3667-3673 (2014).

14. Green DA, Hitoaliaj L, Kotansky B, Campbell SM, Peaper DR. Clinical utility of on-demand multiplex respiratory pathogen testing among adult outpatients. J. Clin. Microbiol. 54(12), 2950-2955 (2016).

15. Harper SA, Bradley JS, Englund JA et al. Seasonal influenza in adults and children-diagnosis, treatment, chemoprophylaxis, and institutional outbreak management: clinical practice guidelines of the infectious diseases society of America. Clin. Infect. Dis. 48(8), 1003-1032 (2009).

16. Shane AL, Mody RK, Crump JA et al. 2017 infectious diseases society of America clinical practice guidelines for the diagnosis and management of infectious diarrhea. Clin. Infect. Dis. 65(12), 1963-1973 (2017).

17. Bhatti MM, Boonlayangoor S, Beavis KG, Tesic V. Evaluation of FilmArray and Verigene systems for rapid identification of positive blood cultures. J. Clin. Microbiol. 52(9), 3433-3436 (2014).

18. Ward C, Stocker K, Begum J, Wade P, Ebrahimsa U, Goldenberg SD. Performance evaluation of the Verigene(R) (Nanosphere) and FilmArray(R) (BioFire(R)) molecular assays for identification of causative organisms in bacterial bloodstream infections. Eur. J. Clin. Microbiol. Infect. Dis. 34(3), 487-496 (2015).

19. De Angelis G, Posteraro B, De Carolis E et al. T2Bacteria magnetic resonance assay for the rapid detection of ESKAPEc pathogens directly in whole blood. J. Antimicrob. Chemother. 73(Suppl. 4), iv20-iv26 (2018).

20. Laffler TG, Cummins LL, McClain CM et al. Enhanced diagnostic yields of bacteremia and candidemia in blood specimens by PCR-electrospray ionization mass spectrometry. J. Clin. Microbiol. 51(11), 3535-3541 (2013).

21. Mylonakis E, Clancy CJ, Ostrosky-Zeichner L et al. T2 magnetic resonance assay for the rapid diagnosis of candidemia in whole blood: a clinical trial. Clin. Infect. Dis. 60(6), 892-899 (2015).

22. Nolling J, Rapireddy S, Amburg JI et al. Duplex DNA-invading gamma-modified peptide nucleic acids enable rapid identification of bloodstream infections in whole blood. MBio 7(2), e00345-00316 (2016).

23. Khumalo J, Nicol M, Hardie D, Muloiwa R, Mteshana P, Bamford C. Diagnostic accuracy of two multiplex real-time polymerase chain reaction assays for the diagnosis of meningitis in children in a resource-limited setting. PLoS ONE 12(3), e0173948 (2017).

24. Sails AD, Eltringham G, Valappil M, Waugh S, Saunders D. Comparison of the Luminex NxTAG respiratory pathogen panel and a multiplex in-house real-time PCR panel for the detection of respiratory viruses in symptomatic patients. J. Med. Microbiol. 4(10), 000562 (2017).

25. Babady NE. The FilmArray (R) respiratory panel: an automated, broadly multiplexed molecular test for the rapid and accurate detection of respiratory pathogens. Expert. Rev. Mol. Diagn. 13(8), 779-788 (2013).

26. Huang RS, Johnson CL, Pritchard L, Hepler R, Ton TT, Dunn JJ. Performance of the Verigene(R) enteric pathogens test, Biofire FilmArray gastrointestinal panel and Luminex xTAG(R) gastrointestinal pathogen panel for detection of common enteric pathogens. Diagn. Microbiol. Infect. Dis. 86(4), 336-339 (2016).

27. Popowitch EB, O'Neill SS, Miller MB. Comparison of the Biofire FilmArray RP, Genmark eSensor RVP, Luminex xTAG RVPv1, and Luminex xTAG RVP fast multiplex assays for detection of respiratory viruses. J. Clin. Microbiol. 51(5), 1528-1533 (2013).

28. Harrington SM, Buchan BW, Doern C et al. Multicenter evaluation of the BD max enteric bacterial panel PCR assay for rapid detection of Salmonella spp., Shigella spp., Campylobacter spp. (C. jejuni and C. coli), and Shiga toxin 1 and 2 genes. J. Clin. Microbiol. 53(5), 1639-1647 (2015).

29. Molling P, Nilsson P, Ennefors T et al. Evaluation of the BD Max enteric parasite panel for clinical diagnostics. J. Clin. Microbiol. 54(2), 443-444 (2016).

30. Buss SN, Leber A, Chapin K et al. Multicenter evaluation of the BioFire FilmArray gastrointestinal panel for etiologic diagnosis of infectious gastroenteritis. J. Clin. Microbiol. 53(3), 915-925 (2015).

31. Hebbelstrup Jensen B, Olsen KE, Struve C, Krogfelt KA, Petersen AM. Epidemiology and clinical manifestations of enteroaggregative Escherichia coli. Clin. Microbiol. Rev. 27(3), 614-630 (2014).

32. Hu J, Torres AG. Enteropathogenic Escherichia coli: foe or innocent bystander? Clin. Microbiol. Infect. 21(8), 729-734 (2015).

33. Furuya-Kanamori L, Marquess J, Yakob L et al. Asymptomatic clostridium difficile colonization: epidemiology and clinical implications. BMC Infect. Dis. 15516 (2015).

34. Murphy CN, Fowler RC, Iwen PC, Fey PD. Evaluation of the BioFire FilmArray(R) GastrointestinalPanel in a Midwestern Academic Hospital. Eur. J. Clin. Microbiol. Infect. Dis. 36(4), 747-754 (2017).

35. Nicholson MR, Van Horn GT, Tang YW et al. Using multiplex molecular testing to determine the etiology of acute gastroenteritis in children. J. Pediatr. 176, 50.e2-56.e2 (2016).

36. US Food and Drug Administration. 1510(k) substantial equivalence determination decision summary (2018). www.accessdata.fda.gov/cdrh_docs/reviews/K143653.pdf

37. Dunbar SA, Zhang H, Tang YW. Advanced techniques for detection and identification of microbial agents of gastroenteritis. Clin. Lab. Med. 33(3), 527-552 (2013). 
38. Schmitz J, Sefers SE. Molecular contamination and amplification product inactivation. In: Advanced Techniques in Diagnostic Microbiology. Tang YW, Stratton CW (Eds.) Springer-Nature, NY, USA, 505-526 (2018).

39. US Food and Drug Administration 510(K) substantial equivalence determination decisionmemorandum (2018). www.accessdata.fda.gov/cdrh_docs/reviews/K152579.pdf

40. Andrews D, Chetty Y, Cooper BS et al. Multiplex PCR point of care testing versus routine, laboratory-based testing in the treatment of adults with respiratory tract infections: a quasi-randomised study assessing impact on length of stay and antimicrobial use. BMC Infect. Dis. 17(1), 671 (2017).

41. Brendish NJ, Malachira AK, Armstrong L et al. Routine molecular point-of-care testing for respiratory viruses in adults presenting to hospital with acute respiratory illness (ResPOC): a pragmatic, open-label, randomised controlled trial. Lancet Respir. Med. 5(5), 401-411 (2017).

42. Pierce VM, Hodinka RL. Comparison of the GenMark Diagnostics eSensor respiratory viral panel to real-time PCR for detection of respiratory viruses in children. J. Clin. Microbiol. 50(11), 3458-3465 (2012).

43. Lee CC, McMillin GA, Babic N, Melis R, Yeo KT. Evaluation of a CYP2C19 genotype panel on the GenMark eSensor(R) platform and the comparison to the Autogenomics Infiniti and Luminex CYP2C19 panels. Clin. Chim. Acta 412(11-12), 1133-1137 (2011).

44. GenMarkDx ePlex Specifications (2018). www.genmarkdx.com/wp-content/uploads/2015/07/GNMK_IMC_1044_E_ePlex_Specifications.pdf

45. GenMarkDx ePlex ${ }^{\circledR}$ : The True Sample-to-Answer Solution (2018). www.genmarkdx.com/solutions/systems/eplex-system/

46. GenMarkDx eSensor Technology (2018) www.genmarkdx.com/solutions/technology/esensor/

47. GenMarkDx GenMark Achieves CE Mark for its ePlex ${ }^{\circledR}$ Sample-to-AnswerSystem (2018). http://ir.genmarkdx.com/static-files/5963974f-1a90-414f-97f6-608563ad65a2

48. US Food and Drug Administration. Administration 510(K) substantial equivalence determination decision summary (2018). www.accessdata.fda.gov/cdrh_docs/reviews/K163636.pdf

- The raw signal from the RP panel actually includes separate coronavirus read-outs for each of these four species. A similar situation applies to several other pathogens on the panel, as individually detected PCR targets also exist for particular clades of rhinovirus and adenovirus. Ultimately, though, these data are integrated into a single reported result for each of the viruses; the individual detected target(s) that lead to the composite call are masked to the operator.

49. Azadeh N, Sakata KK, Brighton AM, Vikram HR, Grys TE. FilmArray respiratory panel assay: comparison of nasopharyngeal swabs and bronchoalveolar lavage samples. J. Clin. Microbiol. 53(12), 3784-3787 (2015).

50. Nijhuis RHT, Guerendiain D, Claas ECJ, Templeton KE. Comparison of ePlex respiratory pathogen panel with laboratory-developed real-time PCR assays for detection of respiratory pathogens. J. Clin. Microbiol. 55(6), 1938-1945 (2017).

51. Ruggiero P, McMillen T, Tang YW, Babady NE. Evaluation of the BioFire FilmArray respiratory panel and the GenMark eSensor respiratory viral panel on lower respiratory tract specimens. J. Clin. Microbiol. 52(1), 288-290 (2014).

52. van Rijn AL, Nijhuis RHT, Bekker V et al. Clinical implications of rapid ePlex(R) respiratory pathogen panel testing compared to laboratory-developed real-time PCR. Eur. J. Clin. Microbiol. Infect. Dis. 37(3), 571-577 (2018).

53. Maubon D, Dard C, Garnaud C, Cornet M. Profile of GenMark's ePlex(R) blood culture identification fungal pathogen panel. Expert. Rev. Mol. Diagn. 18(2), 119-132 (2018).

54. GenMarkDx Press Releases (2018). http://ir.genmarkdx.com/press-releases

55. GenMarkDx ePlex Pipeline (2018). https://genmarkdx.com/solutions/panels/eplex-panels/eplex-pipeline 